\title{
Anxiety, depression and PTSD among children and their parent during 2019 novel coronavirus disease (COVID-19) outbreak in China
}

\author{
Jinming Yue ${ }^{1} \cdot$ Xueyan Zang ${ }^{1} \cdot$ Yunying Le $^{2} \cdot$ Yuanyuan $\mathrm{An}^{1}$ (D) \\ Accepted: 11 November 2020 / Published online: 14 November 2020 \\ (C) Springer Science+Business Media, LLC, part of Springer Nature 2020
}

\begin{abstract}
Home quarantine may lead to families developing a variety of psychological distress. The purpose of this study was to examine the psychological status of children and their parent during 2019 coronavirus disease (COVID-19) outbreak in China. Data were collected from children $(n=1360)$ and their parent $(n=1360)$ in China using online survey during February 2020. Demographic information, media exposure, and psychological status including anxiety, depression and posttraumatic stress disorder (PTSD) symptoms were assessed using self-report measures. The results indicated that, for children, $1.84 \%$ experienced moderate anxiety, $2.22 \%$ experienced depression and $3.16 \%$ met the diagnostic criteria for PTSD; for parent, $1.18 \%, 0.01 \%$ and $3.60 \%$ experienced moderate anxiety, severe depression, and moderate depression, respectively, and $3.53 \%$ met the diagnostic criteria for PTSD. Moreover, excessive media exposure $(\beta=-0.08 \sim 0.13, p \mathrm{~s}<0.05)$ was a risk factor for anxiety and PTSD for children, a positive factor against anxiety and depression for parent. Being a mother $(\beta=0.07 \sim 0.21, p \mathrm{~s}<0.01)$, being younger $(\beta=-0.09$ $\sim-0.07, p \mathrm{~s}<0.05)$, lower levels of educational attainment $(\beta=-0.17 \sim-0.08, p \mathrm{~s}<0.01)$ and family monthly income $(\beta=-0.17$ $\sim-0.11, p \mathrm{~s}<0.05$ ) were risk factors for anxiety, depression and PTSD for parent. Findings suggested that children and their parent in non-severe area didn't suffer major psychological distress during the outbreak. Factors associated with lower levels of mental health problems were identified to inform the use of psychological interventions to improve the mental health of vulnerable groups during the pandemic.
\end{abstract}

Keywords Anxiety $\cdot$ Depression $\cdot$ PTSD $\cdot$ Families in China $\cdot$ Home quarantine

\section{Introduction}

The 2019 coronavirus disease (COVID-19) outbreak is the largest outbreak of atypical pneumonia since the severe acute respiratory syndrome (SARS) outbreak in 2003, and it has quickly become a global health threat (Wang et al. 2020a). Since the outbreak, to retard the spread of the virus, the Chinese government has implemented unprecedentedly strict quarantine measures, with schools at all levels, as well as nonessential businesses, shut down indefinitely. Since January 31th, 2020, the date of returning to work and school has been repeatedly delayed. Social isolation, a very well-documented precursor of mental health problems, could lead to anxiety

Yuanyuan An

anran220@163.com

School of Psychology, Nanjing Normal University, Nanjing, China

2 Department of Psychology, University of Miami, Coral Gables, FL, USA disorders (Barger et al. 2014), major depressive disorder (Lim et al. 2016), and even suicidal behaviors (Calati et al. 2019). Indeed, previous studies revealed profound and wide range of psychological impacts during the COVID-19 outbreak. For example, a study of the general population in isolation found that almost $35 \%$ of the sample experienced psychological distress (Qiu et al. 2020). In another study, the incidences of anxiety, depression and posttraumatic stress disorder (PTSD) in self-isolating general population were $14.4 \%$, $29.7 \%$ and $5.6 \%$, respectively (Zhao et al. 2020).

Among the general population, we should pay special attention to children and their parents as they are particularly vulnerable to the psychological impact of the outbreak (Wang et al. 2020). During the outbreak, children and their parents were confined together at home, and most families stayed socially isolated. As a result, there were substantial changes in their daily routine and social infrastructure. Given quarantine and isolation have undoubtedly affected many aspects of children's and parents' lives, the odds of negative outcomes may increase. Moreover, previous work by Sprang and Silman (2013) found that pandemic disaster and subsequent 
disease-containment measures such as quarantine can be traumatizing to a significant portion of children and parents. Therefore, the current study focuses on the psychological status of these isolated families in China during the COVID-19 outbreak.

With a nationwide school closure, the emergency home schooling plan has been implemented and online courses were offered to children in a well-organized manner by schools and teachers. Although these measures ensured that school learning was not largely disrupted, prolonged school closure and home quarantine might have negative impacts on children's mental health (Brazendale et al. 2017; Brooks et al. 2020), as previous work suggested that children experienced anxiety and depression when facing flexible and rapidly changing situation (Dalton 2020; Tamsin et al. 2020). Indeed, a recent study showed that children suffered greater psychological impact of the outbreak and experienced psychological crisis, which result in a variety of psychological problems such as anxiety and depression (Wang et al. 2020). In Sprang and Silman's study, $30 \%$ of isolated children met the diagnostic criteria for PTSD based on parental reports (Sprang and Silman 2013).

Like their children, parents were confined at home and couldn't go to work and were dealing with a lot of stressors, including the financial loss, the need for basic supplies and heightened concerns for the health of their family members. All of these stressors led to an increased incidence of mental health problems (Reger et al. 2020), and may trigger anxiety, depression and PTSD. In a study during COVID-19 outbreak in Spain, $32.4 \%, 44.1 \%$ and $37.0 \%$ of adults experienced anxiety, depression and psychological stress respectively (Odriozola-González et al. 2020). Similarly, adults in China also reported high levels of psychological distress during the outbreak (Wang et al. 2020b). Furthermore, there was some evidence indicating differential psychological impacts on children and their parents in the context of pandemic disasters and isolation. For example, previous work found that children reported lower levels of psychological distress such as anxiety and depression than their parents (Qiu et al. 2020), but higher prevalence of PTSD (Sprang and Silman 2013). Thus, it is important to take a family perspective and collect data from both children and their parents to further examine and compare their psychological adjustments during the COVID-19 outbreak.

In the face of health-related pandemic disasters like COVID-19, what are the associated factors of mental health problems for children and parents? Individual demographic factors such as gender, age, and educational attainment may have important implication of their psychological status in the face of the pandemic. For example, there was evidence demonstrating higher levels of psychological distress for females than males; those who were younger reported less serious psychological stress; and people with higher education levels tended to be more distressed (Qiu et al. 2020). In addition, media exposure may play a role in people' psychological adjustment. During the health crisis, media become the major source of information for people, and people rely on the media to obtain accurate and up-to-date information in order to make informed decisions about whether they participate in health protective behaviors (Garfin et al. 2020). Research had suggested that repeated media exposure could lead to increased anxiety and heightened stress responses, which can further lead to downstream effects on mental health (Garfin et al. 2020; Thompson et al. 2017). During the outbreak, children and parents in isolation were likely under excessive media exposure with official and non-official information about the COVID-19, which may put them at a greater risk of suffering from anxiety, depression and PTSD. Indeed, it was found that people exposed to excessive media coverage of such kind of traumatic events had even more acute stress than those who were directly exposed to the traumatic events (Giummarra et al. 2018). Therefore, it is important to consider the role of demographics and media exposure on the mental health of children and their parents during the pandemic.

To date, emerging studies have investigated the psychological stress in the general population in China during the COVID-19 outbreak (Qiu et al. 2020; Wang et al. 2020b). However, to our knowledge, no prior study has investigated the psychological impact of COVID-19 from a family's perspective. Thus, the present study is the first to examine the psychological status among families in China facing the COVID-19 outbreak and the associated risk and positive factors.

\section{Method}

\section{Participants}

Cluster sampling was used and participants in the current study were from 1360 families in Jiangsu province, China, including children $(n=1360)$, and their parent $(n=1360)$. Children were on average 10.56 years old $(S D=1.79)$, and 734 (53.97\%) were boys and 626 (46.03\%) were girls; parents were on average 37.78 years old $(S D=4.99)$, with 802 (58.97\%) fathers and 558 (41.03\%) mothers.

\section{Measures}

\section{The Self-Rating Anxiety Scale (SAS)}

Children's and their parent's anxiety symptoms were measured using the Chinese version of Zung Self-rating Anxiety Scale (SAS; Zung 1971). The SAS consists of 20 self-report items assessing both psychological and somatic symptoms of anxiety. Participants rate each item based on their experiences 
within the past week using a 4-point Likert scales ranging from 1 ("none or a little of the time") to 4 ("most or all of the time"). Total raw scores range from 20 to 80 . The standard score was the integer part after the raw score multiplying by 1.25, and higher standard scores indicate higher levels of anxiety symptoms. According to the results of the Chinese norm, the cut-offs score of anxiety is 50, 50-59 suggests mild anxiety, 60-69 suggests moderate anxiety, and 69 and above suggests severe anxiety. In the present study, the Cronbach's $\alpha$ of the scale was 0.81 for children and 0.84 for their parent.

\section{Center for Epidemiologic Studies Depression Scale for Children (CES-DC)}

Children's depression was measured using the Chinese version of Center for Epidemiologic Studies Depression Scale for Children (Fendrich et al. 1990; Wang 1993). It is a 20item self-report measure assessing emotional, cognitive, and behavior related symptoms of depression. For each item, participants were instructed to rate the frequency of each symptom during the past week on a 4-point scale ranging from 0 ("not at all") to 3 ("a lot"). Total possible scores range from 0 to 60 , with higher scores indicating higher levels of depressive symptoms. The cut-offs score for depression is 15 for children. Previous studies have provided evidence of good reliability and construct validity of this measure in various Chinese population (Li et al. 2010; Ying et al. 2013). In the present study, the Cronbach's $\alpha$ of the scale for children was 0.87 .

\section{The Self-Rating Depression Scale (SDS)}

Parent's depression was measured using the Chinese version of Zung Self-rating Depression Scale (Zung et al. 1965), which has been shown to be a valid measure for the Chinese population (Lee et al. 1994). The SDS consists of 20 selfreport items identified using factor analysis. Participants rated each item based on their experiences within the past week using a 4-point Likert scales ranging from 1 ("none or a little of the time") to 4 ("most or all of the time"). Total raw scores range from 20 to 80 . The standard score was the integer part after the raw score multiplying by 1.25 with higher standard scores indicating higher levels of depressive symptoms. According to the results of the Chinese norm, the cut-offs score of depression is 53, 53-62 suggests mild depression, 63-72 suggests moderate depression, and 73 and above suggests severe depression. In the present study, the Cronbach's $\alpha$ of the scale for parent was 0.86 .

\section{PTSD Checklist for DSM-5 (PCL-5)}

Children's and parent's PTSD were assessed using the revised Chinese version of PTSD Checklist for DSM-5
(Weathers 2013; Zhou et al. 2017). All four PSTD symptoms clusters (i.e., "intrusions", "avoidance", "negative cognition and emotion alteration", and "hyper-arousal") were assessed with 20 items. Participants rated the incidence and frequency of their PTSD after a traumatic event on a 4-point Likert scale ranging from 0 ("never") to 3 ("always"). According to the diagnostic criteria for PTSD (Weathers 2013), individuals were considered to be at a "high risk of suffering from PTSD" when all the following four conditions were met at the same time: (1) at least one item's score in the intrusion subscale is equal to or greater than $2 ;(2)$ at least one item's score in the avoidance subscale is equal to or greater than 2; (3) at least two items' scores in the negative cognition and emotion alteration subscale are equal to or greater than 2; (4) at least two items' scores in the hyper-arousal subscale are equal to or greater than 2 . In the present study, the Cronbach's $\alpha$ of the scale was 0.93 for children and 0.93 for their parent.

\section{Media Exposure}

To measure media exposure, children and their parent were instructed to respond to two aspects (i.e., "Subjective media exposure" and "Time of media exposure"). Participants rated two items based on their experiences over the past week (i.e., "The degree of attention paid to information related to the COVID-19 outbreak" and "The time you spent reading and watching news on the COVID-19 outbreak'). A 5-point Likert scale was used ranging from 1 (not at all/ within an hour) to 5 (very much /more than five hours).

\section{Demographic Information}

Sociodemographic variables assessed were children's age and gender; parent's age, gender, educational attainment ("none", "primary school", "middle school," and "bachelor's degree or above") and monthly income ("below 5000RMB", "500010,000RMB”, “10,000-15,000RMB," and "above 15,000RMB').

\section{Procedures}

Data collection took place from February 13th to February 29th, 2020, during which all children and their parent were staying at home because of the COVID-19 pandemic and the unprecedented quarantine order. A total of 1891 children and 2741 parents completed online questionnaires respectively by an APP "Wenjuanxing". We matched children's questionnaires with their parent's questionnaires, and we excluded these invalid questionnaires if either child or his/her parent in a family did not fill out the questionnaire. Finally, 1360 valid questionnaires from children and their parent were used 
in the analysis. The recovery rates of children's and their parent's questionnaires were $71.9 \%$ and $49.6 \%$.

This study was approved by the Ethics Committee of the School of Psychology, Nanjing Normal University. The informed-consent forms were provided to children and their parent, and informed consent was obtained from all participants. At the beginning of the survey, participants were informed about the purposes of this study. Children and their parent completed online questionnaires separately within 15 minutes. After completing the questionnaire, children and their parent were offered three one-hour-long online psychoeducation lessons.

\section{Statistical Analysis}

Data collected in this study were analyzed using SPSS 22.0, and there was no missing data among answers of all data included for the analysis. Descriptive statistics and independent sample T tests of anxiety, depression, and PTSD between children and their parent were conducted. In addition, unary linear regression was used to examine the extent to which each of the demographic variables and media exposure were associated with anxiety, depression and PTSD for children and their parent.

\section{Results}

\section{The Prevalence and Differences between Children and Their Parent in Anxiety, Depression and PTSD}

According to the cut-offs and classification of scoring established for SAS in Zung (1971), 5.66\% children and $4.41 \%$ parents experienced mild anxiety, and $1.84 \%$ children and $1.18 \%$ parents experienced moderate anxiety. Moreover, based on the cut-offs and classification of scoring established for SDS in Zung et al. (1965), 22.79\% parents were mildly depressed, $3.60 \%$ were moderately depressed and $0.01 \%$ were severely depressed. The prevalence of depression for children was $2.22 \%$ using the cut-offs criteria for CES-DC (Fendrich et al. 1990). Finally, using the diagnostic criteria for PTSD
(Weathers 2013), 3.16\% children and 3.53\% parents were identified as at high risk of PTSD.

Furthermore, there were significant differences between children's and their parent's levels of anxiety, depression and PTSD (see Table 1). Different scales were used when measuring depression in children and their parent. Specifically, children experienced significantly higher levels of anxiety $(t=14.53, p<0.001)$, and lower levels of depression $(t=-77.08, p<0.001)$ and PTSD $(t=-7.36, p<0.001)$ than their parent.

\section{Associations of Demographic Variables and Media Exposure with Anxiety, Depression and PTSD}

Results from the regression analyses were presented in Table 2. For children, the risk of anxiety and PTSD was greater for those who spent more time on COVID-19 related media reports $\left(\beta_{\text {anxiety }}=0.07, \beta_{\mathrm{PTSD}}=0.13, p \mathrm{~s}<0.05\right)$, whereas more subjective media exposure $\left(\beta_{\mathrm{PTSD}}=0.13, p<0.001\right)$ only increased the risk of PTSD. For parent, the risk of anxiety, depression and PTSD was greater for those who were women $\left(\beta_{\text {anxiety }}=0.21, \beta_{\text {depression }}=0.12, \beta_{\mathrm{PTSD}}=0.07, p \mathrm{~s}<0.01\right)$, and those with lower educational attainment $\left(\beta_{\text {anxiety }}=\right.$ $\left.-0.16, \beta_{\text {depression }}=-0.17, \beta_{\mathrm{PTSD}}=-0.08, p \mathrm{~s}<0.01\right)$ and lower family monthly income $\left(\beta_{\text {anxiety }}=-0.11, \beta_{\text {depression }}=-0.17\right.$, $\left.\beta_{\mathrm{PTSD}}=-0.11, p \mathrm{~s}<0.001\right)$. Moreover, the risk of anxiety and depression was also greater for parent who were younger $\left(\beta_{\text {anxiety }}=-0.09, \beta_{\text {depression }}=-0.07, p \mathrm{~s}<0.05\right)$ and were under less subjective media exposure $\left(\beta_{\text {anxiety }}=-0.08, \beta_{\text {depression }}=\right.$ $-0.06, p \mathrm{~s}<0.05$ ), whereas spending more time on COVID-19 related media reports $\left(\beta_{\text {anxiety }}=-0.08, p<0.01\right)$ increased the risk of anxiety for parent.

\section{Discussion}

With the ongoing quarantine nationwide, this study was conducted to assess the psychological status of families in China during the COVID-19 outbreak including children and their parent. Factors that predicted the psychological impacts were also explored. Data were collected when children and their parents were quarantined at home. By February 29th, 2020,
Table 1 Descriptive Statistics, Prevalence and the Difference between Children and Their Parent in Anxiety, Depression and PTSD

\begin{tabular}{|c|c|c|c|c|c|c|c|}
\hline Variables & Groups & $n$ & $M$ & $S D$ & Prevalence $(\%)$ & $t$ & $95 \% \mathrm{CI}$ \\
\hline \multirow[t]{2}{*}{ Anxiety } & Children & 1356 & 29.57 & 6.77 & 7.50 & \multirow[t]{2}{*}{$14.53^{* * * *}$} & \multirow[t]{2}{*}{ (3.59 to 4.71 ) } \\
\hline & Parent & 1356 & 25.42 & 8.06 & 5.59 & & \\
\hline \multirow[t]{2}{*}{ Depression } & Children & 1352 & 9.85 & 7.49 & 2.22 & \multirow[t]{2}{*}{$-77.08^{* * * *}$} & \multirow[t]{2}{*}{$(-1.70$ to -1.61$)$} \\
\hline & Parent & 1360 & 35.10 & 9.46 & 26.40 & & \\
\hline \multirow[t]{2}{*}{ PTSD } & Children & 1352 & 7.15 & 7.86 & 3.16 & \multirow[t]{2}{*}{$-7.36^{* * * *}$} & \multirow[t]{2}{*}{$(-2.92$ to -1.69$)$} \\
\hline & Parent & 1358 & 9.46 & 8.46 & 3.53 & & \\
\hline
\end{tabular}

Note. PTSD $=$ Posttraumatic stress disorder; ${ }^{*} p<0.05,{ }^{* *} p<0.01,{ }^{* * * *} p<0.001$ 
Table 2 Linear Regression of Demographic Variables and Media Exposure Predicting Anxiety, Depression and PTSD

\begin{tabular}{|c|c|c|c|c|c|c|c|c|c|c|c|c|}
\hline \multirow[t]{2}{*}{ Variables } & \multicolumn{4}{|c|}{ Anxiety } & \multicolumn{4}{|c|}{ Depression } & \multicolumn{4}{|c|}{ PTSD } \\
\hline & $\Delta \mathrm{R}^{2}$ & $F$ & $\beta$ & $t$ & $\Delta \mathrm{R}^{2}$ & $F$ & $\beta$ & $t$ & $\Delta \mathrm{R}^{2}$ & $F$ & $\beta$ & $t$ \\
\hline \multicolumn{13}{|l|}{ Children } \\
\hline Gender & 0.01 & 1.22 & -0.03 & -1.10 & 0.01 & 0.02 & -0.06 & -0.14 & 0.01 & 0.02 & 0.01 & 0.13 \\
\hline Age & 0.01 & 2.07 & 0.04 & 1.44 & 0.01 & 2.70 & 0.05 & 1.64 & 0.01 & $5.52^{*}$ & 0.06 & $2.35^{*}$ \\
\hline Subjective media exposure & 0.01 & 1.38 & 0.03 & 1.17 & 0.01 & 2.60 & 0.04 & 1.61 & 0.02 & $22.92^{* * *}$ & 0.13 & $4.79^{* * * *}$ \\
\hline Time of media exposure & 0.01 & $6.60^{*}$ & 0.07 & $2.57^{*}$ & 0.01 & 3.39 & 0.05 & 1.84 & 0.02 & $21.87^{* * *}$ & 0.13 & $4.68^{* * * *}$ \\
\hline \multicolumn{13}{|l|}{ Parent } \\
\hline Gender & 0.04 & $59.91^{\text {***** }}$ & 0.21 & $7.74^{* * * *}$ & 0.01 & $19.82^{* * * *}$ & 0.12 & $4.45^{* * *}$ & 0.01 & $7.40^{* * *}$ & 0.07 & $2.72^{* * *}$ \\
\hline Age & 0.01 & $9.48^{* *}$ & -0.09 & $-3.08^{* *}$ & 0.01 & $5.86^{*}$ & -0.07 & $-2.42^{*}$ & 0.01 & 1.40 & -0.03 & -1.18 \\
\hline Educational attainment & 0.03 & $36.69^{* * * *}$ & -0.16 & $-6.06^{* * *}$ & 0.03 & $38.89^{* * * *}$ & -0.17 & $-6.24^{* * *}$ & 0.01 & $8.88^{* * *}$ & -0.08 & $-2.98^{* *}$ \\
\hline Family monthly income & 0.01 & $16.57^{* * *}$ & -0.11 & $-4.07^{* * *}$ & 0.03 & $40.53^{* * *}$ & -0.17 & $-6.37^{* * * *}$ & 0.01 & $16.09^{* * * *}$ & -0.11 & $-4.01^{* * * *}$ \\
\hline Subjective media exposure & 0.01 & $9.06^{* * *}$ & -0.08 & $-3.01^{* *}$ & 0.01 & $4.46^{*}$ & -0.06 & $-2.11^{*}$ & 0.01 & 0.65 & 0.02 & 0.81 \\
\hline Time of media exposure & 0.01 & $7.61^{* *}$ & -0.08 & $-2.76^{* * *}$ & 0.01 & 0.08 & -0.01 & -0.29 & 0.01 & 2.55 & 0.04 & 1.60 \\
\hline
\end{tabular}

Note. PTSD = Posttraumatic stress disorder; ${ }^{*} p<0.05,{ }^{* *} p<0.01,{ }^{* * *} p<0.001$

only a total of 631 confirmed cases and 0 death cases have been reported in Jiangsu province (Jiangsu Commission of Health 2020). Thus, Jiangsu province is classified as one of the non-severely impacted areas. Findings in the current study suggested that children and their parent in non-severe area didn't suffer from major psychological distress during the outbreak (less than $4 \%$ of the respondents experienced moderate or severe levels of psychological problems). This finding is not consistent with findings in previous studies on the psychological impact of health-related pandemic disasters where $30 \%$ of quarantined children and $25 \%$ of their quarantined parents experienced severe psychological trauma (Sprang and Silman 2013). During the time of the data collection, the COVID-19 outbreak is gradually under control. Thus, it is possible that people's psychological distress was gradually alleviated as sense of stability was restored. Moreover, with more and more online mental health services made available to help people quarantined at home, it may also help them better maintain their psychological status and provide strategies to promote positive family interactions (Decosimo et al. 2019; Liu et al. 2020). Besides, home quarantine could provide a good opportunity to foster positive interactions between children and their parent, and such mutual companionship may also to some extent help relieve their psychological distress (Wang et al. 2020c).

In addition, some obvious differences were found in the levels of psychological distress between children and their parent. Specifically, children experienced significantly higher levels of anxiety than their parent, but significantly lower levels of depression and PTSD. First, these differences may be because of the differences in children and their parent's levels of cognitive development. As children's cognitive ability are still developing, they may be more anxious because they have limited understanding of the outbreak and access to coping strategies. Moreover, they may not be able to process all the information they have access to and communicate their feelings like their parents (Imran et al. 2020). Thus, substantial life changes such as the disruption of daily routines and home quarantine may lead to higher levels of anxiety among children than their parent. Parents, on the other hand, have to provide health education for their children while coping with the boredom of quarantine at home and worrying about when they can return to work in an orderly manner, which may explain why parents experienced higher levels of depression and PTSD than their children.

For the purpose of identifying factors associated with the mental health of children and their parent, demographic variables and media exposure in relation to the psychological status of families in China were further explored. First of all, our results suggested that gender and age were predictors of the psychological status of parents, but the same effects were not found for children. The differences in social roles and resource allocation may explain the effect of gender. Parents need to balance their responsibilities for family and work. School closures have undoubtedly increased the needs for childcare to a great extent and the social norms around the gendered division of housework and childcare may explain why the psychological impact were larger for women than men (Alon et al. 2020). Consistently, in another study conducted during the COVID-19 outbreak, women were also found to be at a higher risk of depression and higher levels of psychological stress than men (Wang et al. 2020b). Children were equally stressed and anxious as a result of social isolation, as resources normally available to them through schools were no longer accessible due to school closures (Lee 2020) with little difference across child gender. Moreover, the 
psychological impact on children did not differ across age. This is not consistent with a recent study where younger adults were found to have higher rates of anxiety and depression during outbreaks (Odriozola-González et al. 2020), or other previous studies with similar findings (McManus et al. 2009). Children in the current study were all from primary schools. Thus, the distribution of age is relatively concentrated. To better understand the influence of age on people's psychological status during a pandemic, future studies should include children from a wide age range.

Additionally, it was also found that parents with more family monthly income or higher educational attainment had lower levels of anxiety, depression and PTSD. This is not surprising as previous studies suggested that economic loss caused by home quarantine was a risk factor for anxiety and psychological disorders (Mihashi et al. 2009; Pellecchia et al. 2015). The instability of employment and unemployment brought about by the pandemic crisis and the economic consequences may have less impact on families with higher income (Brooks et al. 2020), as they likely have more savings and resources to cope with the increasing stress brought about by changes in life and work. In addition, educational attainment may serve as a positive factor for anxiety, depression, and PTSD as those with higher educational attainment are better able to obtain and screen information and thus likely have a more comprehensive understanding of the pandemic (Braunack-Mayer et al. 2013; Caleo et al. 2018; Desclaux et al. 2017).

Along with the demographic variables mentioned above, the main channel through which professionals disseminate important information to the public is the media, and repeated exposure to the outbreak related information was found to be closely related to the psychological status of children and their parent. Interestingly, the psychological impact of excessive media exposure was in the opposite direction for children and their parent. Overall, media exposure was a risk factor for anxiety and PTSD for children, but a positive factor against anxiety and depression for parents. This might be due to the differential ability to screen and integrate information. Many recent studies have shown that repeated media exposure and abundance of unsubstantiated information may exacerbate psychological distress in the general public (Brooks et al. 2020; Garfin et al. 2020) and develop into a painful cycle over time such that those who are more concerned about the COVID-19 outbreak may spend more time reading the news each day, which may further increase their stress response (Garfin et al. 2020; Thompson et al. 2019). Compared to their parents, children are less capable of integrating and processing information from the media, and may thus overestimate the threat. Meanwhile, repeated exposure to negative messages can make children anxious about the outbreak. While parents continue to concern about the impact of COVID-19, listening to what children believe about COVID19 transmission and providing children with accurate explanations that are meaningful to them will help alleviate their anxiety (Dalton et al. 2020).

\section{Limitations and Further Research}

First, children in the current study were predominantly in the age range of 8 to 13 years $(n=1256,92.83 \%)$, thus, findings in the current study may not generalize to children of all ages. Previous reviews by Imran et al. (2020) distinguished common symptoms of psychological stress in children of different age groups during the pandemic. The results found that toddlers and preschoolers sense their parents' stress and may display their worries in ways that caregivers may interpret as temper tantrum, aggressive behavior and regressive behaviors, whereas older children and adolescent may feel frustrated, nervous, and bored because of social distancing during this pandemic. We investigated the psychological status of schoolage children during the pandemic, which may help inform psychological interventions in schools. Further research is needed to expand the age range for a more comprehensive understanding of the psychological status of children in all ages during the pandemic to tailor the intervention to further increase the effectiveness. Furthermore, one of the interesting findings that the psychological impact of media exposure was in the opposite direction for children and their parent. This suggested that age may be as moderate factor for the effect of media exposure on psychological status. Children and young people account for $42 \%$ of our world's population, thus, it is important to attend to the immediate and long-term psychological impacts of the COVID-19 outbreak on this population (Dalton et al. 2020). Future research should continue to explore the underlying mechanisms that link demographic variables and media exposure with the psychological adjustments. Another limitation is that we only sampled from Jiangsu province in China. Although our findings provide invaluable information on the psychological status of families in a non-severely impacted area in China during the outbreak, these findings are not generalizable to Chinese families outside of this area, especially families in areas that were severely impacted during the time of the outbreak. Further research is needed to examine and compare the psychological status of children and their parents from areas with varying degrees of severity in order to gain a more systematic understanding of the impact among families during the pandemic in China.

\section{Conclusions}

During the outbreak, children and their parents in nonseverely impacted areas did not suffer major psychological distress, although there were differences between children and their parents in their levels of psychological distress. 
Recent studies suggested that effective communication and positive family interaction provide basis for family members to take care of each other and protect their mental health (Dalton et al. 2020; Szabo et al. 2020). Our findings also identified risk and positive factors for mental health that can be used to inform psychological interventions to improve the mental health of vulnerable groups during the pandemic.

Authors' Contributions Study conception and design, revision: Yuanyuan An.

Acquisition of data: Jinming Yue, Xueyan Zang.

Analysis and interpretation of data: Jinming Yue.

Drafting of manuscript: Jinming Yue, Xueyan Zang.

Critical revision: Yuanyuan An, Yunying Le.

Approved the submitted version for publication: Yuanyuan An.

Funding The study was supported by The Philosophy and Social Science Foundation of Jiangsu (Project No. 18SHC004) and The Natural Science Foundation of Jiangsu Province for Youth (Project No. BK20190702).

\section{Compliance with Ethical Standards}

Competing Interests The author(s) declared no potential conflicts of interest with respect to this research, authorship, and/or the publication of this article.

Code Availability Not applicable.

\section{References}

Alon, T. M., Doepke, M., Olmstead-Rumsey, J., \& Tertilt, M. (2020). The impact of COVID-19 on gender equality (no. w26947). National Bureau of Economic Research. http://www.nber.org/ papers/w26947.

Barger, S. D., Messerli-Bürgy, N., \& Barth, J. (2014). Social relationship correlates of major depressive disorder and depressive symptoms in Switzerland: Nationally representative cross sectional study. BMC Public Health, 14(1). https://doi.org/10.1186/1471-2458-14-273.

Braunack-Mayer, A., Tooher, R., Collins, J. E., Street, J. M., \& Marshall, H. (2013). Understanding the school community's response to school closures during the H1N1 2009 influenza pandemic. BMC Public Health, 13(1), 344. https://doi.org/10.1186/1471-2458-13344.

Brazendale, K., Beets, M. W., Weaver, R. G., Pate, R. R., TurnerMcGrievy, G. M., Kaczynski, A. T., et al. (2017). Understanding differences between summer vs. school obesogenic behaviors of children: The structured days hypothesis. International Journal of Behavioral Nutrition and Physical Activity, 14(1). https://doi.org/10. 1186/s12966-017-0555-2.

Brooks, S. K., Webster, R. K., Smith, L. E., Woodland, L., Wessely, S., Greenberg, N., \& Rubin, G. J. (2020). The psychological impact of quarantine and how to reduce it: Rapid review of the evidence. The Lancet., 395, 912-920. https://doi.org/10.1016/s0140-6736(20) 30460-8.

Calati, R., Ferrari, C., Brittner, M., Oasi, O., Olie, E., Carvalho, A. F., \& Courtet, P. (2019). Suicidal thoughts and behaviors and social isolation: A narrative review of the literature. Journal of Affective Disorders, 15, 653-667. https://doi.org/10.1016/j.jad.2018.11.022.

Caleo, G., Duncombe, J., Jephcott, F., Lokuge, K., Mills, C., Looijen, E., Theoharaki, F., Kremer, R., Kleijer, K., Squire, J., Lamin, M.,
Stringer, B., Weiss, H. A., Culli, D., di Tanna, G. L., \& Greig, J. (2018). The factors affecting household transmission dynamics and community compliance with Ebola control measures: A mixedmethods study in a rural village in Sierra Leone. BMC Public Health, 18(1), 248. https://doi.org/10.1186/s12889-018-5158-6.

Dalton, L., Rapa, E., \& Stein, A. (2020). Protecting the psychological health of children through effective communication about COVID19. The Lancet Child \& Adolescent Health, 4(5), 346-347. https:// doi.org/10.1016/S2352-4642(20)30097-3.

Decosimo, C. A., Hanson, J., Quinn, M., Badu, P., \& Smith, E. G. (2019). Playing to live: outcome evaluation of a community-based psychosocial expressive arts program for children during the Liberian Ebola epidemic. Global Mental Health, 6. https://doi.org/10.1017/gmh. 2019.1.

Desclaux, A., Badji, D., Ndione, A. G., \& Sow, K. (2017). Accepted monitoring or endured quarantine? Ebola contacts' perceptions in Senegal. Social Science \&Medicine, 178, 38-45. https://doi.org/ 10.1016/j.socscimed.2017.02.009.

Fendrich, M., Weissman, M. M., \& Warner, V. (1990). Screening for depressive disorder in children and adolescents: Validating the center for epidemiologic studies depression scale for children. American Journal of Epidemiology, 131, 538-551. https://doi.org/10.1093/ oxfordjournals.aje.a115529.

Garfin, D. R., Silver, R. C., \& Holman, E. A. (2020). The novel coronavirus (COVID-2019) outbreak: Amplification of public health consequences by media exposure. Health Psychology., 39, 355-357. https://doi.org/10.1037/hea0000875.

Giummarra, M. J., Lennox, A., Dali, G., Costa, B., \& Gabbe, B. J. (2018). Early psychological interventions for posttraumatic stress, depression and anxiety after traumatic injury: A systematic review and meta-analysis. Clinical Psychology Review, 62(August 2017), 1136. https://doi.org/10.1016/j.cpr.2018.05.001.

Imran, N., Zeshan, M., \& Pervaiz, Z. (2020). Mental health considerations for children \& adolescents in COVID-19 pandemic. Pakistan Journal of Medical Sciences, 36(COVID19-S4). https:// doi.org/10.12669/pjms.36.COVID19-S4.2759.

Jiangsu Commission of Health (2020). Retrieved March 1, 2020, from http://wjw.jiangsu.gov.cn

Lee, H. C., Chiu, H. F., Wing, Y. K., Leung, C. M., Kwong, P. K., \& Chung, D. W. (1994). The Zung self-rating depression scale: Screening for depression among the Hong Kong Chinese elderly. Journal of Geriatric Psychiatry and Neurology, 7(4), 216-220. https://doi.org/10.1177/089198879400700404.

Lee, J. (2020). Mental health effects of school closures during COVID19. The Lancet. Child \& Adolescent Health., 4, 421. https://doi.org/ 10.1016/S2352-4642(20)30109-7.

Li, W., Cheung, H., Chung, O. K. J., \& Ho, K. Y. (2010). Center for epidemiologic studies depression scale for children: Psychometric testing of the Chinese version. Journal of Advanced Nursing, 66(11), 2582-2591. https://doi.org/10.1111/j.1365-2648.2010. 05440.x.

Lim, M. H., Rodebaugh, T. L., Zyphur, M. J., \& Gleeson, J. F. M. (2016). Loneliness over time: The crucial role of social anxiety. Journal of Abnormal Psychology, 125(5), 620-630. https://doi.org/10.1037/ abn0000162.

Liu, S., Yang, L., Zhang, C., Xiang, Y. T., Liu, Z., Hu, S., \& Zhang, B. (2020). Online mental health services in China during the COVID19 outbreak. The Lancet Psychiatry, 7(4), e17-e18. https://doi.org/ 10.1016/S2215-0366(20)30077-8.

McManus, S., Meltzer, H., Brugha, T., Bebbington, P. E., \& Jenkins, R. (2009). Adult psychiatric morbidity in England: Results of a household survey. Leeds, UK: Health and Social Care Information Centre. https://doi.org/10.13140/2.1.1563.5205.

Mihashi, M., Otsubo, Y., Yinjuan, X., Nagatomi, K., Hoshiko, M., \& Ishitake, T. (2009). Predictive factors of psychological disorder 
development during recovery following SARS outbreak. Health Psychology, 28(1), 91-100. https://doi.org/10.1037/a0013674.

Odriozola-González, P., Planchuelo-Gómez, Á., Irurtia-Muñiz, M. J., \& de Luis-García, R. (2020). Psychological symptoms of the outbreak of the COVID-19 crisis and quarantine in the population of Spain. https://doi.org/10.31234/osf.io/mq4fg.

Pellecchia, U., Crestani, R., Decroo, T., Van den Bergh, R., \& Al-Kourdi, Y. (2015). Social consequences of Ebola containment measures in Liberia. PLoS One, 10(12), e0143036. https://doi.org/10.1371/ journal.pone.0143036.

Qiu, J., Shen, B., Zhao, M., Wang, Z., Xie, B., \& Xu, Y. (2020). A nationwide survey of psychological distress among Chinese people in the COVID-19 epidemic: Implications and policy recommendations. General Psychiatry, 33(2), e100213. https://doi.org/10.1136/ gpsych-2020-100213.

Reger, M. A., Stanley, I. H., \& Joiner, T. E. (2020). Suicide mortality and coronavirus disease 2019-A perfect storm? JAMA Psychiatry., 77, 1093. https://doi.org/10.1001/jamapsychiatry.2020.1060.

Sprang, G., \& Silman, M. (2013). Posttraumatic stress disorder in parents and youth after health- related disasters. Disaster Medicine and Public Health Preparedness, 7(1), 105-110. https://doi.org/10. 1017/dmp.2013.22.

Szabo, T. G., Richling, S., Embry, D. D., Biglan, A., \& Wilson, K. G. (2020). From helpless to hero: Promoting values-based behavior and positive family interaction in the midst of Covid-19. Behavior Analysis in Practice, 1-9. https://doi.org/10.1007/s40617-02000431-0.

Tamsin, F., Tim, V., Katharine, S., Sally, M. M., Anna, G., Salah, M., et al. (2020). Data resource profile: The mental health of children and young people surveys (mhcyp). International Journal of Epidemiology., 49, 363-364g. https://doi.org/10.1093/ije/dyz259.

Thompson, R. R., Garfin, D. R., Holman, E. A., \& Silver, R. C. (2017). Distress, worry, and functioning following a global health crisis: A national study of Americans' responses to Ebola. Clinical Psychological Science, 5(3), 513-521. https://doi.org/10.1177/ 2167702617692030.

Thompson, R. R., Jones, N. M., Holman, E. A., \& Silver, R. C. (2019). Media exposure to mass violence events can fuel a cycle of distress. Science Advances, 5(4), eaav3502. https://doi.org/10.1126/sciadv. aav3502.

Wang, C., Horby, P. W., Hayden, F. G., \& Gao, G. F. (2020a). A novel coronavirus outbreak of global health concern. Lancet, 2020(395), 470-473. https://doi.org/10.1016/s0140-6736(20)30185-9.
Wang, C., Pan, R., Wan, X., Tan, Y., Xu, L., Ho, C. S., \& Ho, R. C. (2020b). Immediate psychological responses and associated factors during the initial stage of the 2019 coronavirus disease (COVID-19) epidemic among the general population in China. International Journal of Environmental Research and Public Health, 17(5), 1729. 1729.https://doi.org/10.3390/ijerph17051729.

Wang, G., Zhang, Y., Zhao, J., Zhang, J., \& Jiang, F. (2020c). Mitigate the effects of home quarantine on children during the COVID-19 outbreak. The Lancet, 395(10228), 945-947. https://doi.org/10. 1016/S0140-6736(20)30547-X.

Wang, X. (1993). Rating scales for mental health (Chinese journal of mental health supplement). Beijing: Chinese Association of Mental Health.

Weathers, F. W. (2013). The PTSD checklist for DSM-5 (PCL-5): Development and initial psychometric analysis. Paper presented at the 29th annual meeting of the International Society for Traumatic Stress Studies, Philadelphia, PA.

Ying, L., Wu, X., Lin, C., \& Chen, C. (2013). Prevalence and predictors of posttraumatic stress disorder and depressive symptoms among child survivors 1 year following the Wenchuan earthquake in China. European Child \& Adolescent Psychiatry, 22, 567-575. https://doi.org/10.1007/s00787-013-0400-3.

Zhao, Y., An, Y., Tan, X., \& Li, X. (2020). Mental health and its influencing factors among self- isolating ordinary citizens during the beginning epidemic of COVID-19. Journal of Loss and Trauma, 25, 114. https://doi.org/10.1080/15325024.2020.1761592.

Zhou, X., Wu, X., \& Zhen, R. (2017). Self-esteem and hope mediate the relations between social support and post-traumatic stress disorder and growth in adolescents following the Ya'an earthquake. Anxiety, Stress, and Coping, 31(1), 32-45. https://doi.org/10.1080/ 10615806.2017.1374376.

Zung, W. W. K. (1971). A rating instrument for anxiety disorders. Psychosomatics: Journal of Consultation and Liaison Psychiatry, 12(6), 371-379. https://doi.org/10.1016/s0033-3182(71)71479-0.

Zung, W. W., Richards, C. B., \& Short, M. J. (1965). Self-rating depression scale in an outpatient clinic: Further validation of the SDS. Archives of General Psychiatry, 13(6), 508-515. https://doi.org/ 10.1001/archpsyc.1965.01730060026004.

Publisher's Note Springer Nature remains neutral with regard to jurisdictional claims in published maps and institutional affiliations. 